\title{
Time-of-day-dependent adaptation of the HPA axis to predictable social defeat stress
}

\author{
C E Koch1,*, M S Bartlang',*, J T Kiehn1, L Lucke3, N Naujokat', C Helfrich-Förster², \\ S O Reber ${ }^{3, *,+}$ and H Oster ${ }^{1, *}$ \\ ${ }^{1}$ University of Lübeck, Chronophysiology Group, Medical Department 1, Lübeck, Germany \\ 2University of Würzburg, Biocenter, Theodor-Boveri-Institute, Neurobiology and Genetics, Würzburg, Germany \\ ${ }^{3}$ Department of Behavioral and Molecular Neurobiology, University of Regensburg, Regensburg, Germany \\ *(C E Koch, M S Bartlang, S O Reber and H Oster contributed equally to this work) \\ †(S O Reber is now at Laboratory for Molecular Psychosomatics, Clinic for Psychosomatic Medicine and \\ Psychotherapy, University of UIm, Ulm, Germany)
}

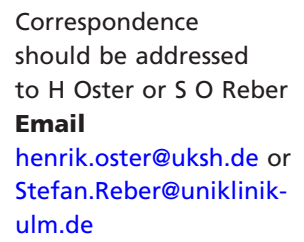

\author{
Key Words \\ - social defeat stress \\ - circadian gating of stress \\ responses \\ - energy homeostasis \\ - HPA axis
}

Journal of Endocrinology (2016) 231, 209-221

\section{Introduction}

In modern societies the rising prevalence of chronic social stress has been suggested to promote the development of various diseases including major depression, cardiovascular diseases and metabolic disorders (Cohen et al. 2007). To understand the underlying mechanisms of stress adaptation and related 
disorders is of high clinical interest. Stress in humans mainly occurs as a consequence of personal interactions or due to a fear of social state loss, for example because of unemployment - factors that are difficult to recreate in experimental animal models (Cohen et al. 2007, Harris 2015). A widely used rodent social stress paradigm is 'repeated social defeat', whereby male animals are repeatedly exposed to subordination by an unknown dominant male conspecific acting as physical and psychosocial stressor (Kudryavtseva et al. 1991, Sgoifo $\&$ Meerlo 2002).

A frequently neglected factor in animal studies that may strongly affect results is the timing of stress interventions. As a response to the earth's rotation around its axis, endogenous circadian clocks have evolved regulating 24-h rhythms of behaviour and physiology, enabling organisms to anticipate and prepare for predictable changes in environmental parameters (for review see Oster et al. (2006)). Ubiquitously expressed cellular oscillators direct tissue-specific transcriptional programmes comprising $5-10 \%$ of the active genome (Panda et al. 2002, Storch et al. 2002). In this way, they organize cellular physiology, but also regulate responses to external stimuli in a time-of-day-dependent fashion (Oster 2006, Lu et al. 2007).

In line with circadian gating of acute stress responses described previously (Zimmermann \& Critchlow 1967, Gibbs 1970, Dunn et al. 1972), we have recently shown that behaviour and immune function of mice predominantly get compromised by repeated social defeat exposure applied during the active, but not during the passive phase (Bartlang et al. 2012, 2014, 2015). In these experiments, repeated social defeat stress during the night was associated with altered circulating stress hormones, while daytime stress had only minor consequences (Bartlang et al. 2012, 2014, 2015).

Within minutes, external stress induces release of glucocorticoids (GCs) (mainly cortisol in humans and corticosterone in rodents) from the adrenal gland to adapt energy metabolism to a perceived fight-or-flight situation. HPA axis activation is initiated by an increase in corticotrophin-releasing hormone (CRH) production in the paraventricular nucleus (PVN) of the hypothalamus. CRH is released into the hypophyseal portal system at the median eminence and transported to the pituitary gland where it promotes adrenocorticotropic hormone (ACTH) secretion into the circulation. $\mathrm{CRH}$ and $A C T H$ expression are further modulated by stress-induced release of arginine vasopressin (AVP) from hypothalamic
PVN and supraoptic nucleus (SON). Through activation of melanocortin-2 receptors (MC2R) in the adrenal cortex ACTH stimulates scavenger receptor B class 1 (SR-B1)mediated uptake of cholesterol esters from blood-borne lipoproteins. A cascade of steroidogenic enzymes converts cholesterol esters into GCs that are directly secreted into the circulation to affect energy metabolism, immune as well as centrally controlled behavioural functions (for review see Spiga et al. (2014)).

In addition to stress, HPA axis activity is regulated by the endogenous circadian clock (Leliavski et al. 2015), at least partly via influencing adrenocortical ACTH sensitivity (Oster et al. 2006, Leliavski et al. 2014). How circadian rhythms affect the adaptive response to repeated and, importantly, predictable stressor exposure, though, remains largely unknown. Therefore, we examined in the current study the impact of 19 days of repeated predictable social defeat stress on HPA axis activity in a time-of-day-dependent manner. In line with previous observations on behaviour and immunity, our data point to a more adaptive response to repeated social stress during the active dark phase. This was indicated by reduced food intake and altered food metabolization associated with adaptations in HPA axis regulation in anticipation of nighttime stressor expose. Exposure to daytime stress, in turn, resulted in much more subtle adaptations, suggesting a circadian gating in the adaptive response to social stress.

\section{Materials and methods}

\section{Mice}

Male C57BL/6 (experimental) and CD1 (resident) mice (obtained from Charles River) were individually housed under standard laboratory conditions at $22 \pm 2^{\circ} \mathrm{C}$, a relative humidity of $60 \pm 5 \%$, a $12 \mathrm{~h}: 12 \mathrm{~h}$ light-darkness cycle (light phase: $200 \pm 50$ lux), and access to food (58\% carbohydrates, $33 \%$ protein, $9 \%$ fat; Ssniff, Soest, Germany) and water ad libitum. Food intake and bodyweight were measured daily between 7 and $8 \mathrm{~h}$ after 'lights on' (Zeitgeber time (ZT) 7-8). At the end of the experiment (on the day after the last social defeat session) mice were killed at the stated time points by $\mathrm{CO}_{2}$ anaesthesia followed by quick decapitation. All experimental protocols were approved by the Committee on Animal Health and Care of the Government of Unterfranken (Bavaria, Germany) and were performed according to international guidelines on the ethical use of animals. 


\section{Social defeat paradigm}

The social defeat paradigm was applied as described previously (Bartlang et al. 2012). Briefly, experimental mice (age: $~ 7$ weeks; bodyweight $20.5 \pm 1.5 \mathrm{~g}$ ) were assigned to a single-housed control (SHC), a daytime social stress (social defeat light, SDL), and a nighttime social stress (social defeat dark, SDD) group in a weight-matched manner (Singewald et al. 2009). Mice of both social stress groups were exposed once daily to social defeat stress for $2 \mathrm{~h}$ over 19 days (except for stress-free days on day 7 and 14), either at the beginning of the light (SDL: ZT1-3) or the beginning of the dark phase (SDD: ZT13-15). During each session, the experimental mouse was placed in the home cage of an aggressive and unfamiliar resident (bodyweight $32.5 \pm 2.5 \mathrm{~g}$ ). After the first attack by the resident resulting in submissive behaviour of the experimental mouse, mice were physically separated by a perforated wall that still allowed visual, olfactory, and auditory contact (Reber et al. 2006). At the end of each defeat session, the partition wall was removed and direct social interaction among the mice was allowed until the first attack by the resident resulting in submissive behaviour of the experimental mouse. After each social defeat session mice were transferred to their individual home cages. To avoid habituation, experimental mice were exposed to a different resident every day.

\section{Central expression of Crh and Avp}

Crh and Avp mRNA levels were measured by in situ hybridization on coronal brain sections as described previously (Reber et al. 2007). 16- $\mu \mathrm{m}$ cryo-sections were collected throughout the extent of the PVN and the SON of brains snap-frozen in dry ice-cooled 2-methylbutane. Hybridization was performed using ${ }^{35 S}$-UTP-labelled antisense RNA probes (sequences: 5'-GGC CCG CGG CGC TCC AGA GAC GGA TCC CCT GCT CAG CAG GGC CCT GCA-3' (Crh) and 5'-GCA GAA GGC CCC GGC CGG CCC GTC CAG CTG CGT GGC GTT GCT CCG GTC-3' (Avp)).

\section{Protein expression}

Protein expression of pituitary CRH receptor 1 (CRHR1), pituitary AVP receptor 1b (AVP-R1b), adrenal MC2R and SR-B1 was determined by Western blot analysis. Tissues were homogenized in EDTA lysis buffer $(100 \mu \mathrm{L}$ for pituitaries, $120 \mu \mathrm{L}$ for adrenals) and rotated at $4^{\circ} \mathrm{C}$ for $1 \mathrm{~h}$. Cellular debris was pelleted by centrifugation $\left(15,700 \mathrm{~g}, 30 \mathrm{~min}, 4^{\circ} \mathrm{C}\right)$. Protein content of supernatants was determined using a BCA Protein Assay Kit (Thermo Scientific). Equal amounts of protein extracts ( $40 \mu \mathrm{g}$ for CRH-R1, $20 \mu \mathrm{g}$ for all other measures) were separated by SDS-PAGE and transferred onto nitrocellulose membranes using a Trans-Blot Electrophoretic Transfer Cell (Bio-Rad). After blocking unspecific protein binding sites with either TBS-T/milk powder (MP) or BSA for $1-2 \mathrm{~h}$, membranes were incubated with specific primary antibody under gentle agitation overnight at $4^{\circ} \mathrm{C}$ (anti-CRH-R1 (V-14) sc-12381 and anti-MC2R (H-300) sc-11412 both 1:200 in 5\% BSA/TBS-T; anti- AVP Receptor V3 (D-20) sc-18105 1:400 in 5\% MP/TBS-T (all from Santa Cruz Biotechnology), anti-SR-B1 ab24603 (Abcam) 1:1600 in $5 \%$ MP/TBS-T and anti- $\beta$-TUBULIN \#2146 (Cell Signaling Technology) 1:1000 in 5\% MP/TBS-T) followed by rinsing and incubation with horseradish peroxidase (HRP) conjugated secondary antibody for $30 \mathrm{~min}$ at RT (for CRH-R1: anti-goat 1:8500; for SR-B1: anti-rabbit 1:3000; for MC2R: anti-rabbit 1:3000; for $\beta$-TUBULIN: anti-rabbit 1:1000). Immunodetection was performed by Enhanced Chemiluminescence (ECL) detection reagent for $1 \mathrm{~min}$ (GE Healthcare). Protein bands were visualized using the Molecular Imager ChemiDoc XRS+ System (Bio-Rad); semi-quantitative densitometric analysis was performed with Image Lab Software (Bio-Rad). Protein expression for each sample was normalized to $\beta$-TUBULIN. For re-use, membranes were stripped by using Re-Blot Plus Mild Antibody Stripping Solution (Millipore).

\section{Adrenal gland and pituitary weight}

Both adrenal glands and the pituitary were dissected from killed mice, cleaned from surrounding fat tissue, weighed and prepared for Western blot analysis (see above).

\section{Lipid droplet staining}

Adrenal lipid droplets were visualized by Oil Red $\mathrm{O}$ staining on $5-\mu \mathrm{m}$ adrenal cryo-sections as described previously (Kudryavtseva et al. 1991). Adrenal sections were fixed for $72 \mathrm{~h}$ in $4 \%$ paraformaldehyde (PFA), washed and rinsed in 60\% isopropyl alcohol for $5 \mathrm{~min}$. Staining was performed for $15 \mathrm{~min}$ with freshly filtered Oil Red O solution (Certistain Oil Red O, Merck) followed by washing in 60\% isopropyl alcohol and distilled water. Covered sections were analyzed using Leica FW 4000 software (Leica Microsystems). 
Adrenal medulla and the zona glomerulosa layer of the cortex were excluded from measurements.

\section{Circulating ACTH and corticosterone}

After 19 days of social defeat stress, mice were killed on day 20 at the predicted time of stressor exposure, but without a previous stress session, either at ZT2 or at ZT14 by $\mathrm{CO}_{2}$ anaesthesia followed by decapitation. Trunk blood was collected in EDTA-coated tubes (Sarstedt, Nürnbrecht, Germany) on ice and centrifuged at $4^{\circ} \mathrm{C}(2300 \mathrm{~g}, 10 \mathrm{~min})$. Plasma ACTH and corticosterone concentrations were analyzed by ELISA (IBL International, Hamburg, Germany).

\section{Excreted corticosterone}

To assess 24-h profiles of GC regulation while avoiding handling, anaesthesia or decapitation effects, we determined the rhythmic excretion of GC metabolites in faeces. Mice were adapted to grid cages for 5 days followed by faeces collection as described previously (Cavigelli et al. 2005, Abraham et al. 2006). Starting one day after the last social stress session, faeces were collected in 4-h intervals for $48 \mathrm{~h}$ (days 20-21). Excreted corticosterone was extracted by boiling powdered faeces in ethanol for $20 \mathrm{~min}$ followed by centrifugation (900 rcf at RT). Ethanol was vaporized at $50^{\circ} \mathrm{C}$ and corticoids were resolved in $1 \mathrm{ml}$ methanol followed by quantification using the Anti-Corticosterone-125I Radioimmunoassay Kit (MP Biomedicals, Orangeburg, NY, USA). This assay was chemically validated in rats previously (Cavigelli et al. 2005), showing that the antibody mainly detects the GC metabolites tetrahydrocorticosterone, 11-dehydrocorticosterone and 21-hydroxy-5 $\beta$-pregnane3,11,20-trione, the excretion rhythm of which closely mimics circadian blood rhythms, although with a delay of several hours (Abraham et al. 2006, Oster et al. 2006). Of note, very low cross-reactivity of the antibody has been reported for other steroids such as desoxycorticosterone $(0.34 \%)$, testosterone $(0.1 \%)$ and cortisol $(0.05 \%)$.

\section{Microdialysis}

Mice were anaesthetized with $2 \%$ isoflurane and a custom-made microdialysis probe (molecular cutoff: $18 \mathrm{kDa})$ was stereotaxically implanted within the right dorsal hippocampus (from bregma: $2.0 \mathrm{~mm}$ lateral, $2.3 \mathrm{~mm}$ posterior, $2.3 \mathrm{~mm}$ ventral). To avoid post-surgical infections, mice received $0.1 \mathrm{~mL}$ of Baytril (2.5\%; Bayer). Correct location of the probe was confirmed after decapitation with a cresyl violet staining

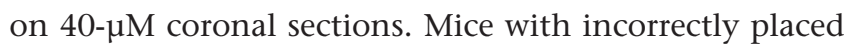
probes were excluded from further analyses. After implantation probes were flushed, filled with sterile Ringer's solution ( $\mathrm{pH}$ 7.4, Braun, Melsungen, Germany), and connected to Ringer-filled polyethylene tubes. On the following day, mice were handled and familiarized with the experimental procedure. After generation of a membrane equilibrium with Ringer's solution $(3.3 \mu \mathrm{L} / \mathrm{min}$ flow rate, $\mathrm{pH} 7.4$, starting at ZT0) measurements were performed on day 22. Samples were collected at two 60-min intervals from ZT2-4 (before acute 5-min swim stress), from ZT6-8 (directly after swim stress) and from ZT8-10. For quantification, dialysates of each $2 \times 60$-min interval pair were pooled, lyophilized, dissolved in $15 \mu \mathrm{L}$ phosphate-buffered saline and corticosterone levels analyzed by ELISA (IBL International). As recovery rates are usually less than $100 \%$ and may vary among probes, we calculated changes in free hippocampal corticosterone of each mouse to the respective baseline value at ZT2-4.

\section{Statistics}

Statistical comparisons were performed with SPSS (version 21.0). Depending on the number of variables, normally distributed data sets were either compared by $t$-test or repeated-measures ANOVA followed by Bonferroni post-hoc tests. Data are presented as mean \pm s.E.M. and considered as significantly different with $P \leq 0.05$. Detailed statistical information are provided in the figure legend of each experiment.

\section{Results}

\section{Chronic social stress affects bodyweight development and food intake}

In line with our previous observations, repeated social defeat stress over 19 days (interrupted by stress-free days on days 7 and 14) either during the light (SDL) or the dark phase (SDD) had only transient effects on bodyweight development in mice (Bartlang et al. 2012). Control (SHC) as well as stressed mice (independent of timing) significantly gained weight during the course of the experiment. The bodyweight of control mice (SHC) increased by $2.9 \pm 0.3 \mathrm{~g}$. Mice subjected to repeated daytime stress $(\mathrm{SDL})$ gained 3.6 $\pm 0.3 \mathrm{~g}$ and mice exposed

Published by Bioscientifica Ltd 

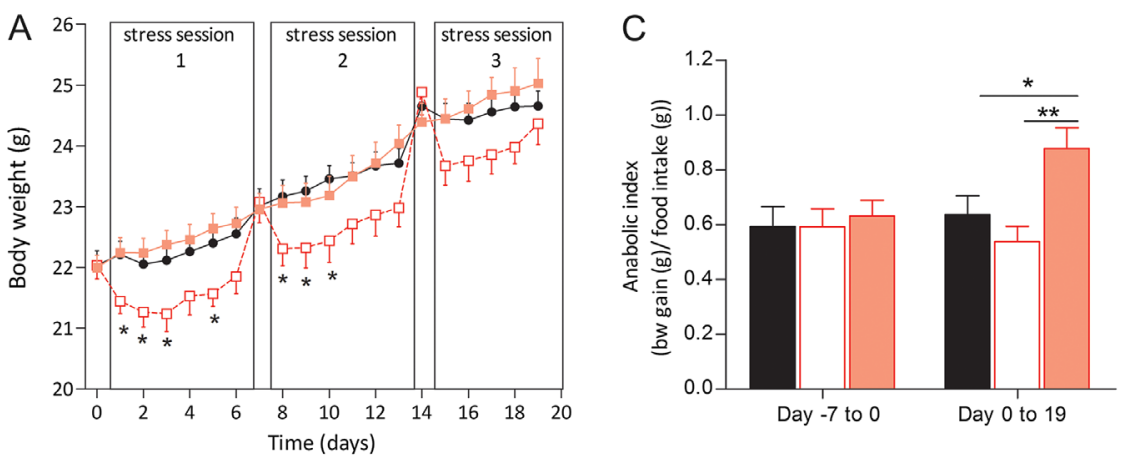

Figure 1

Bodyweight regulation and daily food intake across 19 days of day- vs nighttime social defeat stress. (A) Mice exposed to daily social defeat stress during the night at ZT13-15 (SDD) gain bodyweight similar to single-housed control (SHC) mice. In contrast, mice exposed to social defeat stress during the day at ZT1-3 (SDL) show significantly lower bodyweight on stress days than controls. On stress-free days, SDL mice regain weight, catching up with controls. (B) Cumulative food intake over the course of the experiment is comparable between SHC and SDL and significantly elevated when compared with SDD mice. (C) Comparison of anabolic indices (total bodyweight gain (g)/daily food intake (g)) under pre-stressed conditions (day -7 to 0 ) and over the whole course of the experiment (day 0 to 19). Energy-to-body mass conversion is significantly increased in SDD mice, while anabolic indices of mice exposed to chronic daytime stress (SDL) and controls (SHC) are unchanged. (D) 24-h food intake after the first SD exposure was comparable in all three experimental groups. On stress-free days, SDL mice have a slightly increased food intake, while food intake of SDD mice is reduced. SHC: single-housed controls ( $(\mathbf{0})$; SDL: social defeat light $(\square)$; SDD: social defeat dark $(\square)$; data are presented as means \pm S.E.M.; $n=15 /$ group; ( $A$ and B): repeated measurement two-way ANOVA; (C and $D$ ): one-way ANOVA with Bonferroni post-hoc test; $* P<0.05 ; * * P<0.01 ; * * * P<0.001$.

to stress during the night (SDD) gained $3.4 \pm 0.4 \mathrm{~g}$, without significant differences among groups (Fig. 1A). However, the daytime of stressor exposure differentially affected bodyweight development and food intake (Fig. 1). Social defeat stress during the day (ZT1-3; SDL) led to a transient bodyweight loss reaching its maximum with a loss of $0.8 \pm 0.2 \mathrm{~g}$ after the first 3 days with rapid recovery during stress-free days (Fig. 1A). This transient catabolic effect of daytime stress was repeated in weeks 2 and 3, although to a lower extent, resulting in largely comparable average bodyweight values after 19 days (SHC: $24.7 \pm 0.2 \mathrm{~g}$; SDL: $24.4 \pm 0.3 \mathrm{~g}$; SDD: $25.0 \pm 0.4 \mathrm{~g}$ ). Cumulative food intake of SHC and SDL mice was comparable during the course of the experiment. In contrast, nighttime stress (ZT1315; SDD) led to an overall reduction in food intake (cumulative food intake SHC vs SDD: $P<0.01$; Fig. 1B). During the whole experiment, nighttime stress caused a 40\% increase in the anabolic index (bodyweight gain/ daily food intake) compared with pre-stress conditions (SDD day -7 to 0 (pre-stress): $0.64 \pm 0.1$; SDD day 1 to 19 (stress): $0.88 \pm 0.1 ; P<0.05)$. This effect was absent in control (SHC day -7 to $0: 0.59 \pm 0.1$; $\mathrm{SHC}$ day 1 to 19: $0.64 \pm 0.1$ ) and SDL mice (SDL pre-stress: $0.59 \pm 0.1$;
SDL stress: $0.54 \pm 0.1$; Fig. 1C). The reduction in bodyweight during the first days of social defeat stress intervals in SDL compared with SHC and SDD mice was not associated with reduced food intake. The 24-h food intake on first stress days did not differ among the three groups (Fig. 1D). In line with the observed compensation in bodyweight on stress-free days, SDL mice consumed more food $(4.9 \pm 0.1 \mathrm{~g})$ than SHC (SHC: $4.7 \pm 0.1 \mathrm{~g}$ ) and SDD mice $(4.3 \pm 0.1 \mathrm{~g})$, although significance was only reached against SDD (SDD vs SDL $P<0.001$; SHC vs SDL $P=0.086$; Fig. 1D).

\section{Chronic social nighttime stress (SDD) alters hypothalamic and pituitary stress axis activity at the predicted time of stress}

To characterize the adaptive response of the HPA axis to repeated social defeat stress, we investigated Crh and AVP mRNA expression in PVN and SON as well as CRH-R1 and AVP-R1b protein expression in and ACTH release from the pituitary at the expected time of SD 1 day after the last stressor exposure. Independent of previous stress timing, neither Crh nor AVP transcript 
A

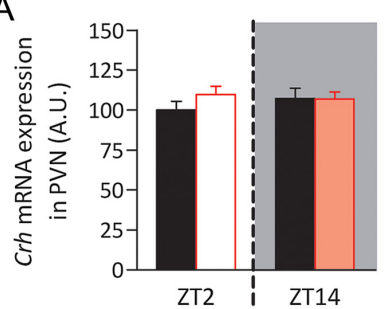

C

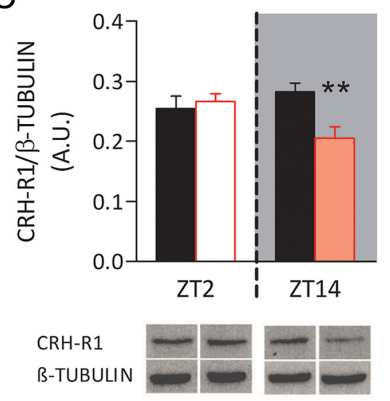

B

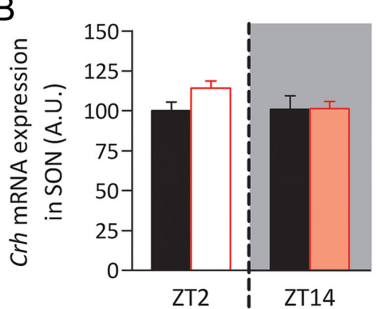

D

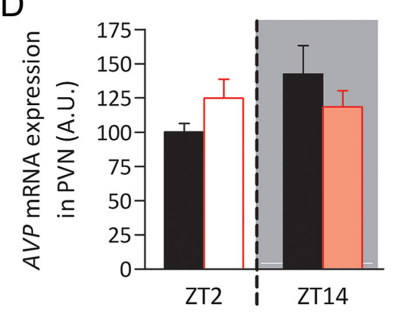

E

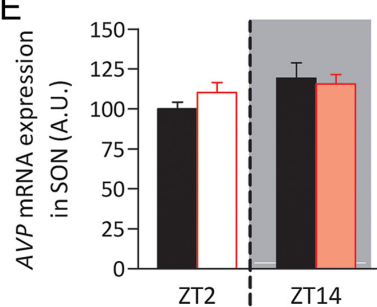

G

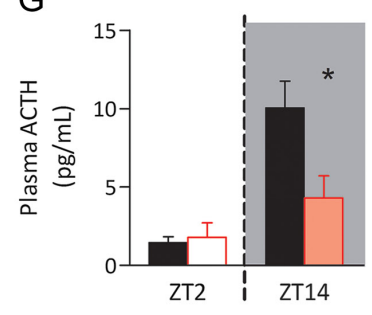

F

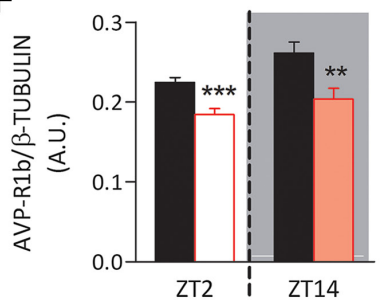

AVP-R1b

B-TUBULIN

$\square \mathrm{SHC} \square \mathrm{SDL} \square \mathrm{SDD}$

\section{Figure 2}

Adaptation of $\mathrm{CRH}$ signalling following repeated social defeat stress. (A, B, D and E) Crh and Avp mRNA expression levels in the PVN (A and D) and the SON (B and E), as assessed by mRNA in situ hybridization on day 20 at the predicted time of stress without previous stressor exposure, were not affected by the stress paradigm. (C and F) Immunoblots for pituitary CRH-R1 and AVP-R1b protein expression reveal a downregulation after repeated social defeat during the night (SDD) compared with controls (SHC) at ZT14 (C and F). Predicted daytime stress does not influence CRH-R1 protein expression at ZT2, but also reduces AVP-R1b protein expression (F). Lower panel: representative blots showing either CRH-R1 or AVP-R1b immunoreactivity together with B-TUBULIN as loading control. (G) Circulating ACTH concentrations at the time of expected SD are significantly reduced in SDD, but not in SDL compared with SHC mice. CRH: corticotrophin-releasing hormone; AVP: arginine vasopressin; PVN: paraventricular nucleus of the hypothalamus; SON: supraoptic nucleus of the hypothalamus; ACTH: adrenocorticotropic hormone; SHC: single-housed controls (O); SDL: social defeat light ( $\square$ ); SDD: social defeat dark $(\square)$; data are presented as mean \pm S.E.M.; $n=6$-9/group; unpaired $t$-tests were used for statistical comparison of SHC and SD mice at specific time points; ${ }^{*} P<0.05 ; * * P<0.01 ; * * * P<0.001$.

levels were significantly altered relative to SHC animals at the expected time of SD exposure (Fig. 2A, B, D and E). However, in the pituitary, CRH-R1 and AVP-R1b protein expression levels were reduced by $\sim 25 \%$ in SDD mice compared with controls (CRH-R1 - SHC: 0.28 \pm 0.01 A.U.; SDD: $\quad 0.21 \pm 0.02$ A.U.; $P=0.006 ; \quad$ AVP-R1b - SHC: $0.26 \pm 0.01$ A.U.; SDD: $0.20 \pm 0.01$ A.U.; $P=0.009$; Fig. $2 \mathrm{C}$ and $\mathrm{F}$ ). Further, circulating ACTH concentrations at this time point were reduced by about $50 \%$ in SDD animals (SHC: $10.1 \pm 1.7 \mathrm{pg} / \mathrm{mL}$; SDD: $4.3 .0 \pm 1.5 \mathrm{pg} / \mathrm{mL}, P=0.024$; Fig. 2D), indicating an attenuated HPA axis activity at the expected time of SD exposure. Preceding social defeat stress during the day had no impact on pituitary CRHR1 protein (SHC: $0.25 \pm 0.02$ A.U.; SDL: $0.27 \pm 0.01$ A.U.; Fig. 2C), but led to a significant reduction in AVPR1b protein expression at ZT2 (SHC: 0.23 \pm 0.01 A.U.; SDL: $\quad 0.18 \pm 0.01$ A.U.; $P=0.001$; Fig. 2F). AVP-R1b suppression in these mice, however, was not associated with reduced circulating ACTH levels at this time point (SHC: $1.4 \pm 0.4 \mathrm{pg} / \mathrm{mL}$; SDL: $1.8 \pm 0.9 \mathrm{pg} / \mathrm{mL}$; Fig. $2 \mathrm{G}$ ). Independent of the previous timing of stressor exposure, no adaptive responses in pituitary weight or the amount of ACTH-positive cells in the pituitary were observed at the time of expected SD (data not shown).

\section{Chronic social nighttime stress (SDD) affects adrenal corticosterone biosynthesis at the predicted time of stress}

In accordance with the observed time-of-day-dependent adaptive response of ACTH secretion to repeated defeat stress, adrenal GC biosynthesis was predominantly affected in SDD mice (Figs 3 and 4). Protein levels of MC2R, the downstream receptor mediating ACTH effects, showed diurnal regulation in control mice with elevated protein expression at ZT14 (SHC). No adaptive response was observed at the time point of expected stressor exposure in both stress groups (ZT2 SHC: $0.12 \pm 0.01$ A.U. SDL $0.14 \pm 0.03$ A.U.; ZT14 SHC: $0.46 \pm 0.03$ A.U.; SDD: $0.42 \pm 0.02$ A.U.; Fig. 3A). SR-B1, an important mediator of adrenal uptake of the GC precursor cholesterol, was expressed at comparable levels in SDL and control animals at the time of expected stress (SHC: $0.47 \pm 0.02$ A.U.; SDL: $0.40 \pm 0.06$ A.U.; Fig. 3B). 
A
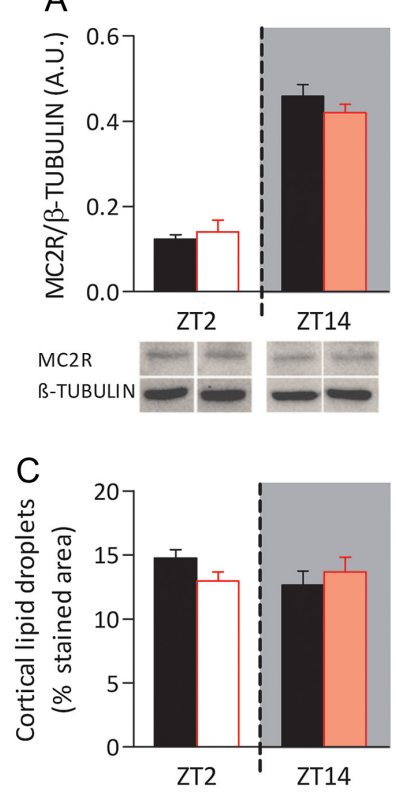

B

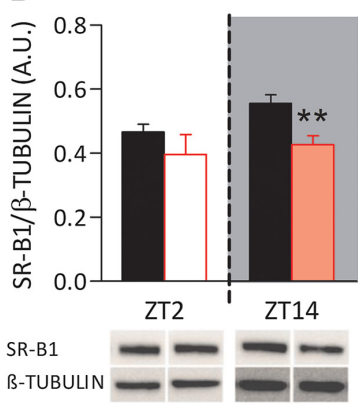

D

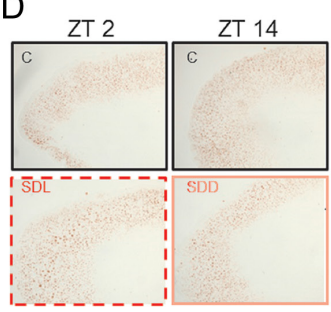

E

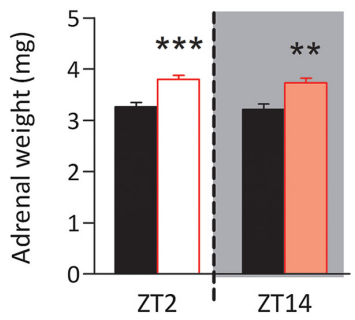

F

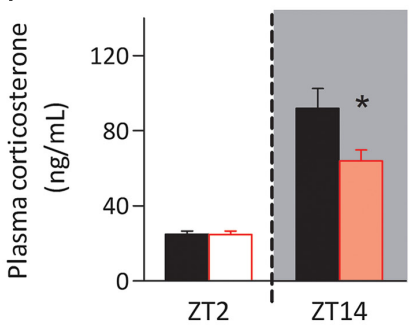

$\square \mathrm{SHC} \square \mathrm{SDL} \square \mathrm{SDD}$

Figure 3

Adaptation of adrenal GC regulation following repeated social defeat stress. (A) MC2R protein levels show a robust diurnal rhythm with elevated expression during the night (ZT14) compared with the day (ZT2) in SHC mice investigated by immunoblots. Preceding daytime defeat stress has no impact on MC2R protein expression. Lower panel: representative blots showing MC2R immunoreactivity and $\beta$-TUBULIN as loading control. (B) 19 days of social defeat stress at night (SDD) led to reduced expression of SR-B1 at ZT14 compared with controls while daytime stress (SDL) does not influence SR-B1 protein expression at the time of expected stress exposure (ZT2). Lower panel: representative immunoblots for SR-B1 and $\beta$-TUBULIN as loading control. ( $C$ and D) Oil-Red O staining of adrenal gland sections does not reveal overt differences in lipid content among stress groups. ( $E$ ) Adrenal gland weights are increased in response to SD independent of the time of stressor exposure. (F) In control animals (SHC), circulating corticosterone concentrations are elevated at ZT14 compared with ZT2. Nighttime levels (ZT14) of circulating corticosterone are decreased after 19 days of chronic nighttime social defeat stress (SDD). Chronic daytime stress (SDL) has no impact on circulating corticosterone concentrations at ZT2, the predicted time of stress. MC2R: melanocortin 2 receptor; SR-B1: scavenger receptor class B, member 1; SHC: single-housed controls (@); SDL: social defeat light ( $\square$ ); SDD: social defeat dark $(\square)$; data are presented as mean \pm S.E.M.; $n=8-9 /$ group; unpaired $t$-tests were used for statistical comparison of SHC and SD mice at specific time points; $* P<0.05 ; * * P<0.01 ; * * * P<0.001$.

However, preceding SD lead to an adaptive decrease in adrenal SR-B1 expression in SDD mice relative to controls at ZT14 (SHC: $0.56 \pm 0.03$ A.U.; SDD: $0.43 \pm 0.03$ A.U.; $P=0.004$; Fig. 3B). It has been shown previously that acute stress can affect adrenal lipid content (Leliavski et al. 2014). Expected defeat stress, however, did not alter adrenal lipid content independent of the time of stressor exposure (ZT2 SHC: $14.8 \pm 0.7 \%$, SDL: $13.0 \pm 0.7 \%$; ZT 14 SHC: $12.7 \pm 1.1 \%$; SDD: $13.7 \pm 1.2 \%$; Fig. $3 \mathrm{C}$ and D). In contrast, adrenal weights were increased in SDD and SDL mice independent of the timing of stress (SHC:

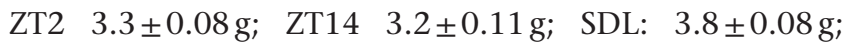
SDD: $3.7 \pm 0.08 \mathrm{~g}$; SHC vs SDL: $P=0.0002$; SHC vs SDD: $P=0.014$; Fig. 3E). Finally, a stress timing-dependent adaptive reduction in the concentration of circulating corticosterone at the expected time of stressor exposure was observed in SDD (SHC: $91.94 \pm 10.6 \mathrm{ng} / \mathrm{mL}$; SDD: $64.07 \pm 5.92 \mathrm{ng} / \mathrm{mL} ; P=0.038$; Fig. $3 \mathrm{~F})$, but not in SDL mice (SHC: $25.08 \pm 1.7 \mathrm{ng} / \mathrm{mL}$; SDL: $24.85 \pm 1.83 \mathrm{ng} / \mathrm{mL}$; Fig. 3F).

\section{Chronic social defeat stress alters diurnal GC release without affecting acute stress responses}

Basal GC release is characterized by a prominent diurnal rhythm peaking at the beginning of the active phase (i.e. the morning in humans, evening in mice (Leliavski et al. 2014)). To investigate whether repeated social defeat would lead to an adaptive response in the circadian regulation of HPA axis activity, we measured faecal corticoid excretion in free-moving mice as an established non-invasive measure of GC rhythms (Cavigelli et al. 2005, Abraham et al. 2006, Oster et al. 2006). As expected, corticoid excretion showed a prominent 24 -h rhythm peaking in the middle of the dark phase in SHC mice (Fig. 4A). After stressor exposure and independent of timing of stressor exposure, faecal corticosterone rhythms were altered (two-way ANOVA factor time $P<0.001$; interaction $P<0.05)$. Consistent with the observed lower circulating corticosterone concentrations at ZT14 (Fig. 3F), excreted corticoid rhythms in SDD mice were altered with lower

Published by Bioscientifica Ltd. 
A

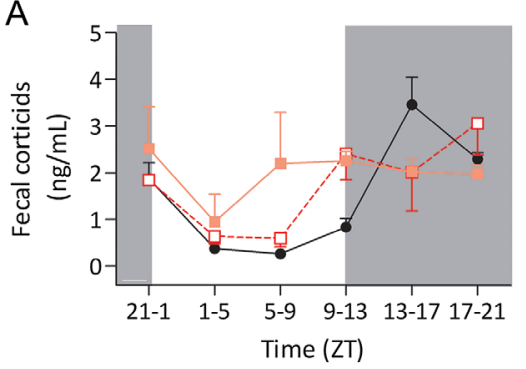

B

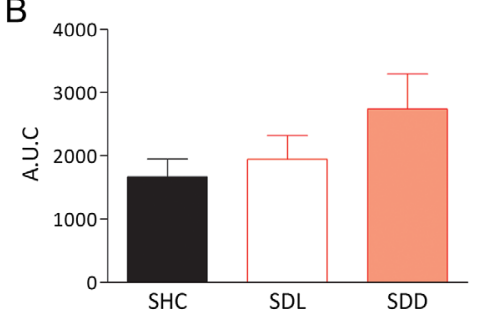

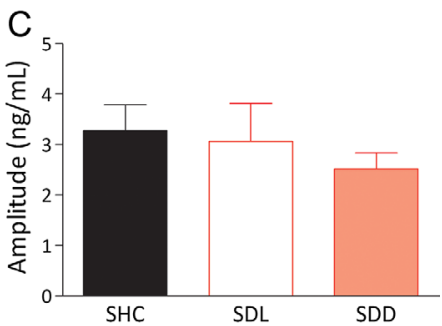
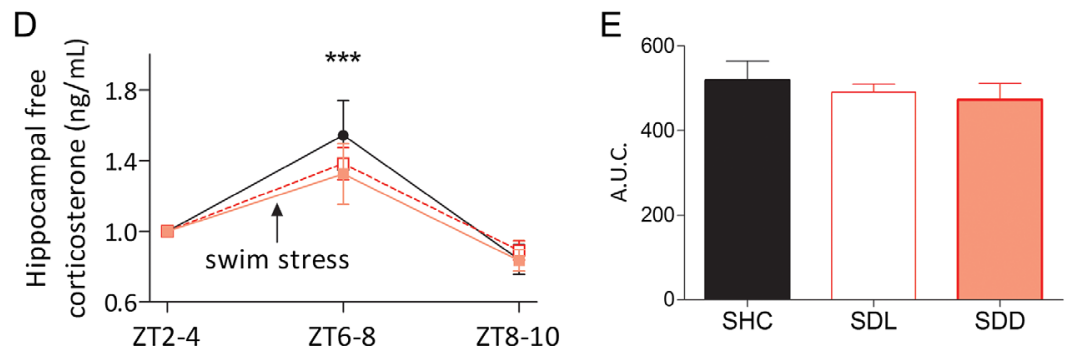

\section{Figure 4}

Effects of repeated social defeat stress on diurnal corticoid excretion rhythms and acute stress responses. (A) Trailing circulating corticosterone levels, excreted corticoids in SHC mice are elevated during the night with a maximum at ZT13-17. This diurnal rhythm is altered in mice exposed to chronic social defeat stress. After SD exposure corticoid levels of SDD and SDL are elevated towards the end of the day and reduced during the night. No significant adaptations are observed for total 24-h excretion (A.U.C.; B) or rhythm amplitude (C). Hippocampal free GC content rises significantly after an acute 5-min swim test in all three groups, independent of prior social stressor exposure. (D) Baseline (ZT2-4), swim stress-induced increase in hippocampal free corticosterone (ZT6-8) and post-stress content (ZT8-10) as well as (E) calculated A.U.C.s were comparable in all three groups. Data are normalized to baseline values. SHC: single-housed controls ( $($ ); SDL: social defeat light ( $\square$ ); SDD: social defeat dark ( $\square$ ); data are presented as mean \pm S.E.M.; A-C: $n=3-4 /$ group, D, E: $n=7$; A, D: repeated measurement two-way ANOVA; B, C, E: one-way ANOVA; $* * * P<0.001$ (relative to ZT2-4).

levels in the second half of the dark phase, while daytime faecal corticoids were elevated at ZT5-9 and ZT9-13 in SDD and SDL mice (Fig. 4A). Total 24-h corticoid excretion (AUC SHC: $1667 \pm 280$; SDD: $2742 \pm 556$; SDL: $1944 \pm 378$; Fig. 4B) and excretion amplitudes were not significantly altered by repeated stress (SHC: $3.3 \pm 0.5$; SDD $2.5 \pm 0.3$; SDL: $3.1 \pm 0.8$; Fig. 4 C).

Despite this adaptation in basal corticoid excretion rhythms as response to predictable stressor exposure, SD did not affect acute responses in a forced swim paradigm. We used a hippocampal microdialysis approach to longitudinally assess GC stress responses without the need of additional handling in SHC, SDD and SDL mice (Fig. 4D and E). Three days after the last social stress session, basal free hippocampal corticosterone concentrations at ZT2-4 were comparable between controls and SD mice. In response to forced swimming for $5 \mathrm{~min}$ free hippocampal corticosterone concentrations consistently rose up. The swim stress induced an 1.5-fold increase $(1.54 \pm 0.2$ A.U.) in free hippocampal corticosterone. Mice previously exposed to repeated social defeat stress showed an increase by 1.3-1.4-fold (SDL: $1.38 \pm 0.09$ A.U.; SDD: $1.32 \pm 0.17$ A.U.) followed by a decline to 0.8-0.9 A.U. (SHC: $0.84 \pm 0.08$ A.U.; SDL: $0.89 \pm 0.06$ A.U.;
SDD: $0.84 \pm 0.06$ A.U.; Fig. 4D). Calculated AUCs were comparable in controls and SD mice (SHC: $519.6 \pm 44.5$; SDL: $490.6 \pm 19.5$; SDD: $473.2 \pm 38.3$; Fig. $4 \mathrm{D}$ and E) suggesting that acute stress responses were not affected by prior social defeat stress, independent of stress timing.

\section{Discussion}

A number of highly prevalent disorders in modern societies, such as obesity or major depression, are thought to be promoted or amplified by chronic stress (Cohen et al. 2007, Harris 2015). However, due to complex interactions with other factors the extent of stress impact is controversially discussed and stress studies in animals do not necessarily represent the human situation (Harris 2015). As one aspect, not only the type and severity, but also the timing of stressor exposure may strongly influence its physiological and psychological effects (Tsang et al. 2014, Leliavski et al. 2015). In humans, the most frequent and detrimental stress affecting well-being arises from social interactions and existential fears (Cohen et al. 2007, Scheuch 2007, Harris 2015). To mimic this situation in a rodent model, we investigated the adaptive response 
to repeated predictable social defeat stress, revealing a prominent time-of-day effect in stress axis adaptation and metabolic regulation.

\section{Timed stressor application differently affects energy metabolism}

While our earlier observations point to a more detrimental effect of nighttime stress in several parameters (Bartlang et al. 2012, 2014, 2015), metabolic effects were predominantly observed in response to stressor exposure during the inactive phase (Rybkin et al. 1997) or as response to continuous stressor exposure (Kudryavtseva et al. 1991, Keeney \& Hogg 1999, Wang et al. 2013). Stress during the active (night) phase did not affect bodyweight gain in rats (Gorka \& Adamik 1993), pointing to a circadian stress response gating. In this study, a direct comparison of repeated daytime versus nighttime stress also revealed that only SDL caused a transient bodyweight loss on stress days measured after stressor exposure, which was not associated with reduced food intake on those days. However, this stress-induced weight loss was quickly compensated on stress-free days, leading to comparable bodyweight at the end of the experiment (Bartlang et al. 2012). In an earlier paper we reported that SDL results in altered diurnal activity patterns and increased body temperature in the early morning (Bartlang et al. 2015). In line with this, it was recently shown in rats that mild chronic subordination leads to reduced activity, altered sleeping patterns and increased body temperature, which suggests an increased metabolic rate as response to SDL (Ahnaou \& Drinkenburg 2016), which is also reported in squirrels (Johren et al. 1991, Kramer et al. 1999). In line with Gorka and Adamik (1993), SDD did not influence bodyweight, but was accompanied by a reduction in food intake, pointing to an anabolic effect of stress during the active phase that is compensated by a reduction in energy intake. However, for a better understanding of the impact of SD on energy metabolism and its anabolic properties it will be important for further studies to characterize changes in energy expenditure and the impact on food digestion as response to expected SDD versus SDL.

\section{Circadian gated adaptation of the HPA axis induced by feedback suppression}

Studies conducted in the 1970s show that the responsiveness of the HPA axis to environmental stimuli varies over the course of the day and strongly depends on the type of stressor (Zimmermann \& Critchlow 1967, Gibbs 1970, Dunn et al. 1972, Kalsbeek et al. 2003). Several aspects of HPA function show circadian variation and, thus, have the potential to promote such time-of-day effects (Barclay et al. 2012). In contrast to the archetypical acute stressinduced activation of the HPA axis, this study provides evidence that repeated predictable stressor exposure at the beginning of the dark phase (SDD) leads to an adaptive response with consistently downregulated HPA axis activity at the predicted stress time (without acute stressor exposure). Circulating ACTH and corticosterone in SDD mice were significantly reduced at ZT14, while plasma concentrations of mice exposed previously to SDL were not altered at ZT2, the predicted time of SD exposure. Interestingly, in our previous study with the same design, SDD caused an increase in circulating plasma ACTH and corticosterone concentrations during the day (ZT5), while nighttime concentrations (ZT17) were unaffected (Bartlang et al. 2012). The corticoid excretion data from this study provide a possible explanation for this apparent discrepancy. Nighttime stress induces a phase shift in diurnal HPA axis activity with reduced activity at the beginning of the dark phase (at the predicted stress time), but elevated activity (and, thus, corticoid excretion) during the light phase (Fig. 4A). Given that corticosterone, via negative feedback inhibition, downregulates HPA axis activity, lower stress hormone secretion in SDD mice at the beginning of the dark phase is likely to be mediated by increased GC concentrations during the day. Similar to SDD, repeated SDL also increased circulating ACTH during the light phase (ZT5) (Bartlang et al. 2012); however, in contrast to SDD, this had no adaptive effect on circulating corticosterone and excreted corticoids at the investigated time point (Figs 3F and 4A).

The reduction in circulating stress hormones at the predicted time of stress in SDD, but not in SDL mice, suggests a circadian clock-gated adaptation of the HPA axis in response to repeated predictable stressor exposure. Although physiological adaptation to repeated psychosocial stress has been reported in humans (Gerra et al. 2001, Wust et al. 2005), the current view generally argues against an adaptation to social defeat stress in rodents (Tornatzky \& Miczek 1993). In line with this, our finding of an unaffected general stress response to an unpredicted and unknown stressor (forced swim stress) in SDD and SDL mice (Fig. 4D) suggests a maintenance of acute HPA axis functionality and a preservation of stress sensitivity after repeated SD. Retained stress responsiveness, however, are

Published by Bioscientifica Ltd. 
contrasted by physiological HPA axis adaptation under non-stressed conditions. Consistent with this we just recently provided evidence for HPA axis adaptation in response to another chronic psychosocial stress paradigm (Uschold-Schmidt et al. 2012).

\section{Downregulation of HPA axis activity in SDD mice}

To reveal the mechanisms underlying the daytimespecific effects of predicted SD on HPA axis activity, we investigated adaptive responses at different levels of the HPA axis at the predicted time of stressor exposure. ACTH release is centrally regulated via hypothalamic $C R H$ and AVP expression (Girotti et al. 2009). While in some chronic stress paradigms hypothalamic CRH expression is elevated (Imaki et al. 1991, Sawchenko et al. 1993, Aguilera 1994, Makino et al. 1995, Albeck et al. 1997) and paralleled by increased circulating ACTH concentrations; other studies report either no stress-induced alterations in hypothalamic $C R H$ expression or even a decrease as a result of chronic stress accompanied by increased AVP expression (Hatalski et al. 1998, Choi et al. 2006, Reber et al. 2007). In our study, Crh as well as Avp transcription in the whole PVN were not affected by repeated nighttime stressor exposure at the expected time of stress, suggesting that lower ACTH levels in SDD mice are not mediated by a decreased PVN input to the pituitary. Of note, since in the mouse, it is technically difficult to differentiate between parvocellular and magnocellullar PVN cells, it is possible that adaptations in the stress responsiveness of parvocellular cells are masked by a lack of stress responsiveness of magnocellular cells. Further, due to the study design we cannot exclude changes in diurnal amplitude or phasing of hypothalamic Crh or Avp expression rhythms that might affect circulating ACTH. In line with the unaffected expression in the PVN, the SON, although did not show the typical stress-induced upregulation of AVP (Ueta et al. 2011, Zhao \& Ai 2011, Steinman et al. 2015). However, consistent with the idea that SDD affects HPA axis downstream of the PVN, pituitary CRH-1R and AVP-R1b expression were reduced by expected SDD.

Repeated stress has been shown to affect not only ACTH release, but also the adrenal's ACTH sensitivity (Bartlang et al. 2012, Uschold-Schmidt et al. 2012) and capacity to mobilize cholesterol (Uschold-Schmidt et al. 2012, Fuchsl et al. 2013). While adrenal MC2R protein expression was unaffected by SD in our study, the highdensity lipoprotein (HDL) receptor SR-B1 (Kraemer 2007), was downregulated specifically in SDD mice, suggesting a decreased SR-B1-mediated uptake of HDL-bound cholesterol resulting in reduced GC biosynthesis at the time of predicted stress. Hoekstra et al. have shown that adrenal-specific SR-B1 deletion causes GC insufficiency (Hoekstra et al. 2013). Given that adrenal SR-B1 expression is regulated by ACTH signalling (Rigotti et al. 1996), the adaptive downregulation of SR-B1 in SDD is likely to be at least partly mediated by reduced circulating ACTH levels. Further studies, however, are needed to dissect the underlying mechanisms. At this point, we cannot exclude stress-mediated alterations in MC2R signalling as only receptor expression - and not activity or localization was investigated.

In contrast to the more consistent adaptive responses of HPA axis activity to SDD, SDL led to less extensive physiological adaptations only at the level of the pituitary at the predicted stress time. Neither hypothalamic nuclei nor adrenal glands show adaptations at the predicted time of stressor exposure. This, together with our previous findings suggesting a more detrimental effects of SDD in general (Bartlang et al. 2012, 2014, 2015), supports the idea that social stress responses are gated by the circadian system leading to a time-of-day-dependent plasticity of the HPA axis. However, since the impact of SD was only investigated at the predicted time of stress, we cannot exclude adaptive alterations at other times of day or general changes in the circadian regulation of the HPA axis.

\section{Metabolic relevance of HPA axis adaptations}

Based on the data from our short-term study it is difficult to predict, if and how the observed adaptive changes in the HPA axis regulation in response to repeated social stress may contribute to long-term alterations in energy intake and bodyweight regulation. It is well established that ACTH and GCs influence adipose physiology (Feldman \& Loose 1977, Cavigelli et al. 2005, Campbell et al. 2011, Lee et al. 2014). Moreover, circadian clocks in adipose tissue respond to GC treatment (Shostak et al. 2013a) and have been shown to affect food intake and energy homeostasis via regulation of fatty acid release from adipose stores (Paschos et al. 2012, Shostak et al. 2013a,b, Kolbe et al. 2015). In addition, nighttime stress modulates central pacemaker function (Bartlang et al. 2014) and thereby can promote internal circadian misalignment, which has been associated with obesity and metabolic impairments (Karlsson et al. 2001, Barclay et al. 2012). Therefore, the

Published by Bioscientifica Ltd 
circadian gating of stress effects may modulate energy metabolism. The dissection of the underlying pathways warrants further studies.

\section{Conclusion}

In summary, our results indicate that adaptive responses to repeated psychosocial stressor exposure are strongly dependent on the time of day when the stressor is applied. While repeated daytime social defeat stress had transient catabolic effects without strongly altering HPA axis activity at the investigated time point, predicted nighttime social defeat had plastic effects on HPA axis regulation at the level of the pituitary and the adrenal, resulting in a dampened diurnal GC rhythm. Our previous studies have indicated that repeated stressor exposure during the active, but not inactive, phase goes along with decreased activity, social deficits and intestinal inflammation. Our new data suggests that circadian gating may be an underestimated factor in assessing the impact of chronic stressors on general well-being.

\section{Declaration of interest}

The authors declare that there is no conflict of interest that could be perceived as prejudicing the impartiality of the research reported.

\section{Funding}

This study was supported by the Deutsche Forschungsgemeinschaft (DFG FO-207/13-1, OS-223/03-1 \& TR-SFB 134) and a Lichtenberg Fellowship of the Volkswagen Foundation ( $\mathrm{HO}$ ). The funders had no role in study design, data collection and analysis, decision to publish or preparation of the manuscript.

\section{Acknowledgements}

C E Koch, M S Bartlang, S O Reber and H Oster contributed equally to this work.

\section{References}

Abraham D, Dallmann R, Steinlechner S, Albrecht U, Eichele G \& Oster H 2006 Restoration of circadian rhythmicity in circadian clock-deficient mice in constant light. Journal of Biological Rhythms 21 169-176. (doi:10.1177/0748730406288040)

Aguilera G 1994 Regulation of pituitary ACTH secretion during chronic stress. Frontiers in Neuroendocrinology 15 321-350. (doi:10.1006/ frne.1994.1013)

Ahnaou A \& Drinkenburg WH 2016 Simultaneous changes in sleep, qEEG, physiology, behaviour and neurochemistry in rats exposed to repeated social defeat stress. Neuropsychobiology 73 209-223. (doi:10.1159/000446284)

Albeck DS, McKittrick CR, Blanchard DC, Blanchard RJ, Nikulina J, McEwen BS \& Sakai RR 1997 Chronic social stress alters levels of corticotropin-releasing factor and arginine vasopressin mRNA in rat brain. Journal of Neuroscience 17 4895-4903.

Barclay JL, Husse J, Bode B, Naujokat N, Meyer-Kovac J, Schmid SM, Lehnert H \& Oster H 2012 Circadian desynchrony promotes metabolic disruption in a mouse model of shiftwork. PLOS ONE 7 e37150. (doi:10.1371/journal.pone.0037150)

Bartlang MS, Neumann ID, Slattery DA, Uschold-Schmidt N, Kraus D, Helfrich-Forster C \& Reber SO 2012 Time matters: pathological effects of repeated psychosocial stress during the active, but not inactive, phase of male mice. Journal of Endocrinology 215 425-437. (doi:10.1530/JOE-12-0267)

Bartlang MS, Savelyev SA, Johansson AS, Reber SO, Helfrich-Forster C \& Lundkvist GB 2014 Repeated psychosocial stress at night, but not day, affects the central molecular clock. Chronobiology International $\mathbf{3 1}$ 996-1007. (doi:10.3109/07420528.2014.940085)

Bartlang MS, Oster H \& Helfrich-Forster C 2015 Repeated psychosocial stress at night affects the circadian activity rhythm of male mice. Journal of Biological Rhythms 30 228-241. (doi:10.1177/0748730415576192)

Campbell JE, Peckett AJ, D'Souza AM, Hawke TJ \& Riddell MC 2011 Adipogenic and lipolytic effects of chronic glucocorticoid exposure. American Journal of Physiology: Cell Physiology 300 C198-C209. (doi:10.1152/ajpcell.00045.2010)

Cavigelli SA, Monfort SL, Whitney TK, Mechref YS, Novotny M \& McClintock MK 2005 Frequent serial fecal corticoid measures from rats reflect circadian and ovarian corticosterone rhythms. Journal of Endocrinology 184 153-163. (doi:10.1677/joe.1.05935)

Choi DC, Nguyen MM, Tamashiro KL, Ma LY, Sakai RR \& Herman JP 2006 Chronic social stress in the visible burrow system modulates stress-related gene expression in the bed nucleus of the stria terminalis. Physiology \& Behavior 89 301-310. (doi:10.1016/j. physbeh.2006.05.046)

Cohen S, Janicki-Deverts D \& Miller GE 2007 Psychological stress and disease. JAMA 298 1685-1687. (doi:10.1001/jama.298.14.1685)

Dunn J, Scheving L \& Millet P 1972 Circadian variation in stress-evoked increases in plasma corticosterone. American Journal of Physiology 223 402-406.

Feldman D \& Loose D 1977 Glucocorticoid receptors in adipose tissue. Endocrinology 100 398-405. (doi:10.1210/endo-100-2-398)

Fuchsl AM, Uschold-Schmidt N \& Reber SO 2013 Chronic psychosocial stress in male mice causes an up-regulation of scavenger receptor class B type 1 protein in the adrenal glands. Stress 16 461-468. (doi:10.310 9/10253890.2013.793303)

Gerra G, Zaimovic A, Mascetti GG, Gardini S, Zambelli U, Timpano M, Raggi MA \& Brambilla F 2001 Neuroendocrine responses to experimentally-induced psychological stress in healthy humans. Psychoneuroendocrinology 26 91-107. (doi:10.1016/S03064530(00)00046-9)

Gibbs FP 1970 Circadian variation of ether-induced corticosterone secretion in the rat. American Journal of Physiology 219 288-292.

Girotti M, Weinberg MS \& Spencer RL 2009 Diurnal expression of functional and clock-related genes throughout the rat HPA axis: system-wide shifts in response to a restricted feeding schedule. American Journal of Physiology: Endocrinology and Metabolism 296 E888-E897. (doi:10.1152/ajpendo.90946.2008)

Gorka Z \& Adamik P 1993 The effect of reserpine and stress on feeding behaviour in the light and dark phases of the diurnal cycle in rats. Journal of Pharmacy and Pharmacology 45 137-138 (doi:10.1111/j.2042-7158.1993.tb03698.x)

Harris RB 2015 Chronic and acute effects of stress on energy balance: are there appropriate animal models? American Journal http://joe.endocrinology-journals.org

DOI: 10.1530/JOE-16-0163
() 2016 Society for Endocrinology Printed in Great Britain
Published by Bioscientifica Ltd 
of Physiology: Regulatory, Integrative and Comparative Physiology $\mathbf{3 0 8}$ R250-R265. (doi:10.1152/ajpregu.00361.2014)

Hatalski CG, Guirguis C \& Baram TZ 1998 Corticotropin releasing factor mRNA expression in the hypothalamic paraventricular nucleus and the central nucleus of the amygdala is modulated by repeated acute stress in the immature rat. Journal of Neuroendocrinology $10663-669$ (doi:10.1046/j.1365-2826.1998.00246.x)

Hoekstra M, van der Sluis RJ, Van Eck M \& Van Berkel TJ 2013 Adrenalspecific scavenger receptor BI deficiency induces glucocorticoid insufficiency and lowers plasma very-low-density and low-density lipoprotein levels in mice. Arteriosclerosis, Thrombosis, and Vascular Biology 33 e39-e46. (doi:10.1161/ATVBAHA.112.300784)

Imaki T, Nahan JL, Rivier C, Sawchenko PE \& Vale W 1991 Differential regulation of corticotropin-releasing factor mRNA in rat brain regions by glucocorticoids and stress. Journal of Neuroscience 11 585-599.

Johren O, Topp H, Sander G, Schoch G \& Fuchs E 1991 Social stress in tree shrews increases the whole-body RNA degradation rates. Naturwissenschaften 78 36-38. (doi:10.1007/BF01134043)

Kalsbeek A, Ruiter M, La Fleur SE, Van Heijningen C \& Buijs RM 2003 The diurnal modulation of hormonal responses in the rat varies with different stimuli. Journal of Neuroendocrinology 15 1144-1155. (doi:10.1111/j.1365-2826.2003.01112.x)

Karlsson B, Knutsson A \& Lindahl B 2001 Is there an association between shift work and having a metabolic syndrome? Results from a population based study of 27,485 people. Occupational and Environmental Medicine 58 747-752. (doi:10.1136/oem.58.11.747)

Keeney AJ \& Hogg S 1999 Behavioural consequences of repeated social defeat in the mouse: preliminary evaluation of a potential animal model of depression. Behavioural Pharmacology 10 753-764. (doi:10.1097/00008877-199912000-00007)

Kolbe I, Dumbell R \& Oster H 2015 Circadian clocks and the interaction between stress axis and adipose function. International Journal of Endocrinology 2015 693204. (doi:10.1155/2015/693204)

Kraemer FB 2007 Adrenal cholesterol utilization. Molecular and Cellular Endocrinology 265-266 42-45. (doi:10.1016/j.mce.2006.12.001)

Kramer M, Hiemke C \& Fuchs E 1999 Chronic psychosocial stress and antidepressant treatment in tree shrews: time-dependent behavioral and endocrine effects. Neuroscience and Biobehavioral Reviews 23 937-947. (doi:10.1016/S0149-7634(99)00027-5)

Kudryavtseva NN, Bakshtanovskaya IV \& Koryakina LA 1991 Social model of depression in mice of C57BL/6J strain. Pharmacology, Biochemistry, and Behavior 38 315-320. (doi:10.1016/00913057(91)90284-9)

Lee MJ, Pramyothin P, Karastergiou K \& Fried SK 2014 Deconstructing the roles of glucocorticoids in adipose tissue biology and the development of central obesity. Biochimica et Biophysica Acta 1842 473-481. (doi:10.1016/j.bbadis.2013.05.029)

Leliavski A, Shostak A, Husse J \& Oster H 2014 Impaired glucocorticoid production and response to stress in Arntl-deficient male mice. Endocrinology 155 133-142. (doi:10.1210/en.2013-1531)

Leliavski A, Dumbell R, Ott V \& Oster H 2015 Adrenal clocks and the role of adrenal hormones in the regulation of circadian physiology. Journal of Biological Rhythms 30 20-34. (doi:10.1177/0748730414553971)

Lu HZ, Huang L, So RM \& Wang J 2007 A computational study of the interaction noise from a small axial-flow fan. Journal of the Acoustical Society of America 122 1404. (doi:10.1121/1.2764474)

Makino S, Smith MA \& Gold PW 1995 Increased expression of corticotropin-releasing hormone and vasopressin messenger ribonucleic acid (mRNA) in the hypothalamic paraventricular nucleus during repeated stress: association with reduction in glucocorticoid receptor mRNA levels. Endocrinology 136 3299-3309. (doi:10.1210/ en.136.8.3299)

Oster H 2006 The genetic basis of circadian behavior. Genes, Brain, and Behavior 5 (Supplement 2) 73-79. (doi:10.1111/j.1601183X.2006.00226.x)
Oster H, Damerow S, Kiessling S, Jakubcakova V, Abraham D, Tian J, Hoffmann MW \& Eichele G 2006 The circadian rhythm of glucocorticoids is regulated by a gating mechanism residing in the adrenal cortical clock. Cell Metabolism 4 163-173. (doi:10.1016/j. cmet.2006.07.002)

Panda S, Antoch MP, Miller BH, Su AI, Schook AB, Straume M, Schultz PG, Kay SA, Takahashi JS \& Hogenesch JB 2002 Coordinated transcription of key pathways in the mouse by the circadian clock. Cell 109 307-320. (doi:10.1016/S0092-8674(02)00722-5)

Paschos GK, Ibrahim S, Song WL, Kunieda T, Grant G, Reyes TM, Bradfield CA, Vaughan CH, Eiden M, Masoodi M, et al. 2012 Obesity in mice with adipocyte-specific deletion of clock component Arntl. Nature Medicine 18 1768-1777. (doi:10.1038/nm.2979)

Reber SO, Obermeier F, Straub RH, Falk W \& Neumann ID 2006 Chronic intermittent psychosocial stress (social defeat/overcrowding) in mice increases the severity of an acute DSS-induced colitis and impairs regeneration. Endocrinology 147 4968-4976. (doi:10.1210/en.20060347)

Reber SO, Birkeneder L, Veenema AH, Obermeier F, Falk W, Straub RH \& Neumann ID 2007 Adrenal insufficiency and colonic inflammation after a novel chronic psycho-social stress paradigm in mice: implications and mechanisms. Endocrinology 148 670-682. (doi:10.1210/en.2006-0983)

Rigotti A, Edelman ER, Seifert P, Iqbal SN, DeMattos RB, Temel RE, Krieger M \& Williams DL 1996 Regulation by adrenocorticotropic hormone of the in vivo expression of scavenger receptor class B type I (SR-BI), a high density lipoprotein receptor, in steroidogenic cells of the murine adrenal gland. Journal of Biological Chemistry $\mathbf{2 7 1}$ 33545-33549. (doi:10.1074/jbc.271.52.33545)

Rybkin II, Zhou Y, Volaufova J, Smagin GN, Ryan DH \& Harris RB 1997 Effect of restraint stress on food intake and body weight is determined by time of day. American Journal of Physiology $\mathbf{2 7 3}$ R1612-R1622.

Sawchenko PE, Arias CA \& Mortrud MT 1993 Local tetrodotoxin blocks chronic stress effects on corticotropin-releasing factor and vasopressin messenger ribonucleic acids in hypophysiotropic neurons. Journal of Neuroendocrinology 5 341-348. (doi:10.1111/j.1365-2826.1993. tb00493.x)

Scheuch K 2007 [Disease caused by psychological stress on the job-what is assured?]. Deutsche Medizinische Wochenschrift 132 601-602. (doi:10.1055/s-2007-973598)

Sgoifo A \& Meerlo P 2002 Animal models of social stress: implications for the study of stress related pathologies in humans. Stress $\mathbf{5} 1-2$. (doi:10.1080/102538902900012314)

Shostak A, Husse J \& Oster H 2013a Circadian regulation of adipose function. Adipocyte 2 201-206. (doi:10.4161/adip.26007)

Shostak A, Meyer-Kovac J \& Oster H 2013b Circadian regulation of lipid mobilization in white adipose tissues. Diabetes 62 2195-2203. (doi:10.2337/db12-1449)

Singewald GM, Nguyen NK, Neumann ID, Singewald N \& Reber SO 2009 Effect of chronic psychosocial stress-induced by subordinate colony (CSC) housing on brain neuronal activity patterns in mice. Stress 12 58-69. (doi:10.1080/10253890802042082)

Spiga F, Walker JJ, Terry JR \& Lightman SL 2014 HPA axis-rhythms. Comprehensive Physiology 4 1273-1298. (doi:10.1002/cphy.c140003)

Steinman MQ, Laredo SA, Lopez EM, Manning CE, Hao RC, Doig IE, Campi KL, Flowers AE, Knight JK \& Trainor BC 2015 Hypothalamic vasopressin systems are more sensitive to the long term effects of social defeat in males versus females. Psychoneuroendocrinology $\mathbf{5 1}$ 122-134. (doi:10.1016/j.psyneuen.2014.09.009)

Storch KF, Lipan O, Leykin I, Viswanathan N, Davis FC, Wong WH \& Weitz CJ 2002 Extensive and divergent circadian gene expression in liver and heart. Nature $\mathbf{4 1 7}$ 78-83. (doi:10.1038/nature744)

Tornatzky W \& Miczek KA 1993 Long-term impairment of autonomic circadian rhythms after brief intermittent social stress. Physiology \& Behavior 53 983-993. (doi:10.1016/0031-9384(93)90278-n) 
Tsang AH, Barclay JL \& Oster H 2014 Interactions between endocrine and circadian systems. Journal of Molecular Endocrinology 52 R1-R16. (doi:10.1530/JME-13-0118)

Ueta Y, Dayanithi G \& Fujihara H 2011 Hypothalamic vasopressin response to stress and various physiological stimuli: visualization in transgenic animal models. Hormones and Behavior 59 221-226. (doi:10.1016/j.yhbeh.2010.12.007)

Uschold-Schmidt N, Nyuyki KD, Fuchsl AM, Neumann ID \& Reber SO 2012 Chronic psychosocial stress results in sensitization of the HPA axis to acute heterotypic stressors despite a reduction of adrenal in vitro ACTH responsiveness. Psychoneuroendocrinology 37 1676-1687. (doi:10.1016/j.psyneuen.2012.02.015)

Wang J, Chai A, Zhou Q, Lv L, Wang L, Yang Y \& Xu L 2013 Chronic clomipramine treatment reverses core symptom of depression in subordinate tree shrews. PLOS ONE 8 e80980. (doi:10.1371/journal. pone.0080980)

Wust S, Federenko IS, van Rossum EF, Koper JW \& Hellhammer DH 2005 Habituation of cortisol responses to repeated psychosocial stress-further characterization and impact of genetic factors. Psychoneuroendocrinology 30 199-211. (doi:10.1016/j.psyneuen.2004.07.002)

Zhao DQ \& Ai HB 2011 Oxytocin and vasopressin involved in restraint water-immersion stress mediated by oxytocin receptor and vasopressin $1 \mathrm{~b}$ receptor in rat brain. PLOS ONE $\mathbf{6}$ e23362. (doi:10.1371/journal.pone.0023362)

Zimmermann E \& Critchlow V 1967 Effects of diurnal variation in plasma corticosterone levels on adrenocortical response to stress. Proceedings of the Society for Experimental Biology and Medicine 125 658-663. (doi:10.3181/00379727-125-32172)

Received in final form 24 August 2016

Accepted 22 September 2016

Accepted Preprint published online 22 September 2016 (c) 2016 Society for Endocrinology Printed in Great Britain
Published by Bioscientifica Ltd 